\title{
Altered emotional prosody processing in patients with Parkinson's disease after subthalamic nucleus stimulation
}

This article was published in the following Dove Press journal:

Neuropsychiatric Disease and Treatment

\author{
Yazhou Jin* \\ Zhiqi Mao* \\ Zhipei Ling \\ Xin Xu \\ Guang Xie \\ Xinguang Yu \\ Department of Neurosurgery, \\ People's Liberation Army General \\ Hospital, Beijing, People's \\ Republic of China \\ *These authors contributed equally \\ to this work
}

Background: Patients with Parkinson's disease (PD) exhibit deficits in recognizing and expressing vocal emotional prosody. The aim of this study was to explore emotional prosody processing in patients with PD shortly after subthalamic nucleus (STN) deep brain stimulation (DBS).

Methods: Two groups of patients with PD (pre-DBS and post-DBS) and one healthy control (HC) group were recruited as participants. All participants (PD and HC) were assessed using the Montreal Affective Voices database 50 Voices Recognition test. All participants were asked to nonverbally express five basic emotions (happiness, anger, fear, sadness, and neutral) to test emotional prosody expression. Fifteen native Chinese speakers were recruited as raters. We recorded the accuracy rate, reaction time, confidence level, and two acoustic parameters (mean pitch and mean intensity).

Results: The PD groups scored lower than the HC group in recognizing and expressing emotional prosody. STN DBS had no significant effect on the recognition of emotional prosody but had a significant effect on fear prosody expression. Pearson's correlation analysis revealed significant correlations between performance on emotional prosody recognition tests and performance on emotional prosody expression tests in both the pre-DBS PD and post-DBS PD groups.

Conclusion: Shortly after STN DBS, the ability to recognize emotional prosody was not altered, but fear expression was impaired. We identified associations between abnormalities in emotional prosody recognition and expression deficits both before and after STN DBS, indicating that the processes involved in recognizing and expressing emotional prosody may share a common system.

Keywords: Parkinson's disease, emotional prosody, subthalamic nucleus stimulation, emotional recognition, emotional expression

\section{Introduction}

Deep brain stimulation (DBS) is an established therapy for patients with Parkinson's disease (PD) who have failed to respond to or are not suitable for dopaminergic replacement therapy. DBS has been shown to provide a clear benefit for patients with PD by reducing motor dysfunction. The following three well-established stimulation targets are currently recognized: the subthalamic nucleus (STN), globus pallidus internus (GPi), and pedunculopontine nucleus. ${ }^{1}$ Among these regions, the STN and GPi are the most commonly targeted structures. Comparative studies of these two targets have shown that both have unique advantages and disadvantages. ${ }^{2-4}$ When choosing the most suitable target, factors such as dyskinesia, medication reduction, and nonmotor symptoms should also be included in addition to motor symptoms.
Correspondence: Xinguang Yu

Department of Neurosurgery, People's Liberation Army General Hospital,

28 Fuxing Road, Haidian District,

Beijing, 100853, People's Republic of

China

Tel +8610638939

Email xinguangyu30I@I63.com 
Defects in cognition and vocal expression are two important nonmotor symptoms, and the administration of STN DBS to patients with PD has been shown to induce modifications in symptoms. ${ }^{5,6}$ Deficits in cognition and vocal expression impact emotional recognition and expression in patients with PD. Emotional expression includes the expression of emotional faces and voices. ${ }^{7}$ Compared to facial expression, vocal expression better conveys an individual's inner thoughts. In other words, the way we speak is more important than the content of our speech and emotional prosody communicates one's emotional status.

Segmental and suprasegmental changes occur during the course of pronunciation and affect acoustic parameters, such as speed, pitch, and intensity, and these changes are collectively called emotional prosody. ${ }^{5,8}$ Using functionalmagnetic resonance imaging (MRI) and stereotactic electroencephalography, the neural networks involved in emotional prosody processing have been shown to operate in three stages. ${ }^{9}$ In the first stage, inputs resulting from auditory information activate primary and secondary auditory cortical regions, predominately those in the right hemisphere..$^{10,11}$ The second stage is thought to involve projections from the superior temporal gyrus to the anterior superior temporal sulcus, which contains voice-sensitive neurons. ${ }^{12,13}$ The third stage mainly concerns the processing of vocal emotional perception, which is mediated by projections from the superior temporal sulcus to the orbitofrontal cortex and right inferior frontal gyrus. ${ }^{10,14,15}$ In addition to brain network structures in the fronto-temporal lobe, structures in the limbic system, such as the amygdala, thalamus, and basal ganglia, have been reported to be involved in emotional prosody processing. ${ }^{16-19}$ Recently, a series of studies aimed at exploring STN DBS in emotional prosody recognition suggested that the STN is also a crucial structure for decoding emotional prosody..$^{11,20}$

The ability to recognize and express vocal emotional prosody is damaged in some pre-DBS patients. ${ }^{21,22}$ According to Rusz et $\mathrm{al}^{23}{ }^{23}$ the reduced ability to vocally express emotions is detected even in the early stage of PD. However, researchers have not clearly determined whether these processes are impaired to a greater extent in patients with PD who underwent STN DBS. ${ }^{24,25}$ In fact, most of the studies of emotional prosody processing have only examined one cognitive feature (either recognition or expression), and these studies have yielded controversial results. Although deficits in emotional prosody recognition ${ }^{11,20,26}$ or emotional prosody expression ${ }^{6}$ have been reported to be more severe after STN DBS, others have failed to identify abnormalities..$^{24,25}$ The conflicting results presented in these studies are likely caused by confounding factors (such as a depressive state and cognitive impairment in patients with PD) and differences in methodologies. In addition, further studies are required to determine whether abnormalities in emotional prosody recognition or expression deficits are associated with STN DBS.

The aim of this study was to explore emotional prosody processing in patients with PD shortly after STN DBS. This approach allowed us to evaluate early outcomes in postoperative nonmotor symptoms. In this study, both recognition and expression tests were performed. In addition, subjective and objective methods were used to evaluate emotional prosody expression. According to previous studies, two basic human emotions, such as disgust and surprise, have relatively low recognition rates..$^{27,28} \mathrm{We}$ therefore included the following five basic human emotions in this study: happiness, sadness, fear, anger, and neutral. The results of our study will improve our understanding of the changes that occur in emotional prosody processing in patients with PD who undergo STN DBS.

\section{Participants and methods Participants}

Two groups of patients with PD (pre-DBS and post-DBS) and one group of healthy controls (HCs) were recruited as participants. All three groups were matched for gender, age, and years of education. The pre-DBS group comprised 16 patients with PD (11 men and five women) who were candidates for STN DBS, whereas the post-DBS group comprised 16 patients (11 men and five women) who had already undergone bilateral STN DBS for 6 months. Another 16 healthy individuals (11 men and five women) were recruited as the $\mathrm{HC}$ group. All participants agreed to participate in the study and signed the informed consent form.

The following inclusion criteria were used to recruit patients with PD: a clinical diagnosis of PD; poor motor symptom control when using dopaminergic medications; a good response to a levodopa test; and an absence of cognitive dysfunction (a Montreal Cognitive Assessment score of $>24$ and a Mini Mental State Examination score of $>26$ ), depression (diagnosed according to the Hamilton Depression Scale), anxiety (diagnosed according to the Hamilton Anxiety Scale), and other psychiatric diseases. The HC group was also tested using the same scales to exclude cognitive dysfunction or other psychiatric diseases. In addition, we analyzed the clinical characteristics of two PD groups, such as disease duration, Unified Parkinson's Disease Rating Scale III, Hoehn and Yahr staging scale, and levodopa equivalent daily dose. Group differences in demographic and neuropsychological characteristics were analyzed using one-way analysis of variance (ANOVA). Clinical data were analyzed using independent two-sample Student's $t$-tests. Mann-Whitney $U$ tests were 
Table I Demographic and clinical data

\begin{tabular}{|c|c|c|c|c|c|c|c|}
\hline Variables & $H C(n=16)$ & Pre-PD $(n=16)$ & Post-PD $(n=16)$ & Statistical methods & $d f$ & Statistical values & $P$-values \\
\hline Gender ratio & $5 F / I I M$ & $5 F / I I M$ & $5 F / I I M$ & & & & \\
\hline Age (years) & $61.75 \pm 7.50$ & $61.63 \pm 8.31$ & $61.93 \pm 5.95$ & ANOVA & 2 & 0.396 & 0.993 \\
\hline Education (years) & $8.75 \pm 3.19$ & $8.94 \pm 3.23$ & $8.63 \pm 2.58$ & ANOVA & 2 & 0.043 & 0.957 \\
\hline MMSE & $28.19 \pm 0.91$ & $28.06 \pm 0.85$ & $28.13 \pm 0.89$ & ANOVA & 2 & 0.080 & 0.923 \\
\hline MOCA & $27.00 \pm 0.97$ & $26.19 \pm 1.05$ & $26.56 \pm 1.15$ & ANOVA & 2 & 2.364 & 0.106 \\
\hline Disease duration (years) & NA & $8.06 \pm 2.29$ & $7.56 \pm 1.90$ & Independent sample $t$-test & 30 & 0.672 & 0.507 \\
\hline $\mathrm{H} \& Y$ & NA & $2.50 \pm 0.73$ & $2.44 \pm 0.5 \mathrm{I}$ & Mann-Whitney U & 2 & 127 & 0.965 \\
\hline LEDD & NA & $391.84 \pm 193.11$ & $380.47 \pm 200.4 \mathrm{I}$ & Independent sample $t$-test & 30 & 0.163 & 0.871 \\
\hline UPDRS III & NA & $29.56 \pm 15.15$ & $28.75 \pm 13.79$ & Independent sample $t$-test & 30 & 0.159 & 0.875 \\
\hline
\end{tabular}

Note: Data given are mean \pm SD.

Abbreviations: ANOVA, analysis of variance; F, female; HC, healthy control; H\&Y, Hoehn and Yahr staging scale; LEDD, Levodopa equivalent daily dose; M, male; MMSE, Mini Mental State Examination; MOCA, Montreal Cognitive Assessment; NA, not applicable; PD, Parkinson's disease; UPDRS, Unified Parkinson's Disease Rating Scale.

used if the normality assumptions were not satisfied. The demographic characteristics, neuropsychological characteristics, and clinical data are shown in Table 1.

\section{Neurosurgery}

Under local anesthesia, patients were placed in a stereotactic head frame (Leksell Model F head frame). Then, a 1.5 T scanner (Siemens Espree) was used to perform stereotactic MRI to identify the anterior commissure (AC) and posterior commissure (PC). We obtained a $3 \mathrm{~mm}$ thick axial image parallel to the AC-PC plane. Coronal and sagittal images orthogonal to the axial images were also acquired. Similar to the findings described by Benabid et al, ${ }^{29,30}$ the anatomical target coordinates for the STN were $4 \mathrm{~mm}$ inferior, $3 \mathrm{~mm}$ posterior, and $12 \mathrm{~mm}$ lateral to the midpoint of the AC-PC line. Because individual differences in STN morphology were observed across the patients, the target was adjusted to the center of the STN. The entry points were determined based on MRI images and the target coordinates. DBS 3389 electrodes (Medtronic, Minneapolis, MN, USA) were placed using intraoperative microelectrode recording. Patients remained awake to test the temporary efficacy of the procedure using a temporary external stimulator (Programmer 8840), to obtain verbal feedback, and to avoid unwanted adverse effects. An intraoperative MRI (iMRI) was then performed to confirm the accuracy of the electrode placement. ${ }^{31}$ Once the iMRI showed that the electrodes were positioned in the center of and at an appropriate depth within the STN, a pulse generator (Medtronic) was placed in the chest area under the clavicle using a routine procedure; otherwise, the coordinates were adjusted and the second round of MRI scans was performed to ensure the appropriate location of the electrodes. In our center, we initiate stimulation 2 weeks after hospital discharge. The active stimulation contact is placed in the dorso-lateral part of the STN in each hemisphere. Before applying stimulating pulses, all four contacts of bilateral electrodes were tested.
We regularly set a pulse width of $\sim 60 \mu \mathrm{S}$, a frequency of $\sim 130 \mathrm{~Hz}$, and a voltage of $\sim 1.5 \mathrm{~V}$. The follow-up DBS programming procedures were performed 1 month, 3 months, 6 months, and 1 year later. At these time points, subjects were still undergoing active programming adjustment. In addition, DA medications were still changing. Unlike some centers, the dose reduction occurs more slowly in our center.

We used a single contact of the quadripolar electrode, and monopolar stimulation was performed in all patients. The stimulation characteristics are shown in Table 2.

\section{Procedure}

The experimental procedure consisted of three main experiments. The first experiment focused on the recognition of emotional prosody, the second experiment was concerned with recording expressions of emotional prosody expression, and the third experiment was performed to subjectively and objectively evaluate perceptions of emotional expression. All experiments were conducted in a quiet, softly lit room. During the course of the experimental procedures, the patients in the pre-DBS group were on-dopa whereas the patients in the post-DBS group were on-dopa and on-stimulation.

\section{Experiment I: emotional prosody recognition}

All participants (PD and $\mathrm{HC}$ ) were assessed using the Montreal Affective Voices (MAV) database 50 Voices Recognition test. ${ }^{32}$ The test consisted of 50 nonverbal affect

Table 2 Stimulation parameters for post-PD group

\begin{tabular}{llll}
\hline Sides & Voltage (V) & Pulse (us) & Frequency (Hz) \\
\hline Left & $2.25 \pm 0.41$ & $71.25 \pm 8.85$ & $141.88 \pm 19.74$ \\
Right & $2.16 \pm 0.49$ & $70.63 \pm 9.29$ & $144.38 \pm 21.59$ \\
\hline
\end{tabular}

Note: Data given are mean \pm SD

Abbreviation: PD, Parkinson's disease. 
bursts that corresponded to the emotions, such as happiness, anger, fear, sadness, and neutral, and recorded from 10 different actors (five males and five females). These voices were played randomly and one at a time. Then, the participants were asked to carefully listen to each voice at a comfortable volume while wearing headphones and to identify the correct emotion categories using a five forced-choice response format. Although the reaction time (RT) was not limited, the participants were also encouraged to make their choices quickly. The recognition test provided scores with a maximum value of 10 for each single emotion and yielded a score with a maximum of 50 for all five emotions. Before the test, the participants were asked to describe each type of emotion to obtain a better understanding of the prosody recognition test.

All 50 recognition trials were run in E-Prime 2.0 software. Each trial sequence consisted of 1) the presentation of a fixation cross at the center of a computer screen for $1,000 \mathrm{~ms}$, 2) an audio presentation of an exemplar over a loudspeaker along with the simultaneous display of an image, and 3) a display that presented the five forced-choice response formats.

\section{Experiment 2: emotional prosody expression}

Similar to the MAV database used in experiment 1, all participants were asked to nonverbally express five basic emotions (including happiness, anger, fear, sadness, and neutral). A high-quality microphone was placed $\sim 15 \mathrm{~cm}$ from the participant's mouth. Voices were recorded using the Adobe Audition 3.0 software (Adobe, Inc., San Jose, CA, USA) and edited into sound files in waveform audio format format. The length of the sound files corresponding to each type of emotion was 1 second. Before each recording, a 6 second blank section was recorded for subsequent noisereduction processing. All recordings were performed under the same conditions, using consistent recording parameters (a sampling rate of $48,000 \mathrm{~Hz}$, mono channel, and a resolution ratio of 16 bits). To ensure that the participants understood the task, an instruction was provided, such as "What do you say when you feel happiness, sadness, fear, anger, and a neutral emotion?"

\section{Experiment 3: emotional prosody perception}

Both subjective and objective methods were used to determine whether the materials recorded in experiment 2 conveyed the intended emotions.
In the subjective method, 15 native Chinese speakers (eight males and seven females) were recruited to validate the recorded materials. They were all graduate students at the People's Liberation Army General Hospital with normal hearing and no diseases. The students lived in China, and their native language was Chinese. The average age was 27.26 years $(\mathrm{SD}=1.53)$. The average number of years of education was 20.33 ( $\mathrm{SD}=1.13$ ). All participants in this experiment volunteered to participate in the study without payment, and they were blind to the patients' diagnoses. All recorded materials were divided into three groups (such as two PD groups and one $\mathrm{HC}$ group). Each voice was randomly played and one at a time. The participants in this experiment were asked to sit in front of a computer screen and carefully listen to each recording at a comfortable volume level while wearing headphones. Then, they were asked to distinguish the correct emotion category using a five-choice format (happiness, anger, fear, sadness, and a neutral emotion). In addition, they were required to rate their confidence level (CL) regarding their choices (zero points, none; one point, very little; two points, a little; three points, mild; four points, high; and five points, very high). The accuracy rates and RTs of the participants were also calculated. All trials testing perception were performed using the E-Prime 2.0 software. Inter-rater reliability was assessed by calculating Cronbach's alpha.

In the objective method, two acoustic parameters (mean pitch and mean intensity) of the recorded materials were analyzed to explore whether the parameters are useful for distinguishing emotional speech across the three groups. ${ }^{33,34}$ A total of 240 emotional speech materials were included. Emotional speech was analyzed using the Praat speech analysis software package (available at: www.praat.org [Phonetic Sciences, University of Amsterdam, the Netherlands]). ${ }^{35}$ For the entire duration of each speech material, the following two acoustic parameters were included: mean pitch in hertz and mean intensity in decibels. An author (GX) verified the results of automatic analysis to ensure the accuracy of the automatic procedure.

\section{Ethical aspects}

The ethics committee of the People's Liberation Army General Hospital approved the research protocol.

\section{Statistical analysis}

Total MAV scores were analyzed using ANOVA. In addition, MAV subscores were assessed using ANOVA with a $3 \times 5$ design (between-subject factors: pre-DBS, post-DBS, 
and $\mathrm{HC}$; within-subject factors: happiness, anger, fear, sadness, and neutral). Analogous statistical analyses were designed to evaluate the accuracy rate of the perception of vocal expressions. Two $3 \times 5$ designs (between-subject factors: pre-DBS, post-DBS, and $\mathrm{HC}$; within-subject factors: happiness, anger, fear, sadness, and neutral) were performed for the variables RT and CL in the perception of vocal expression. For the objective method, mean pitch and mean intensity were separately compared using an ANOVA with a $3 \times 5$ design (between-subject factors: pre-DBS, post-DBS, and $\mathrm{HC}$; within-subject factors: happiness, anger, fear, sadness, and neutral). Post hoc least significant difference (LSD) tests were applied to identify differences between groups. Tests for normality and homogeneity of variance were also performed before each variance analysis. The data were analyzed using $\chi^{2}$ tests instead of ANOVA if the normality and homogeneity of variance assumptions were not satisfied. Post hoc Mann-Whitney $U$ tests were used to identify differences between groups.

The correlations between performance on tests of recognition and expression in each group were assessed using the Pearson correlation test. Additional Pearson's correlation tests were performed to analyze correlations between performance on expression tests and the two acoustic parameters (mean pitch and mean intensity). Spearman's correlation tests were used if the normality assumptions were not satisfied.

All statistical analyses were performed using the SPSS 17.0 software package. $P$-value $\leq 0.05$ was considered statistically significant.

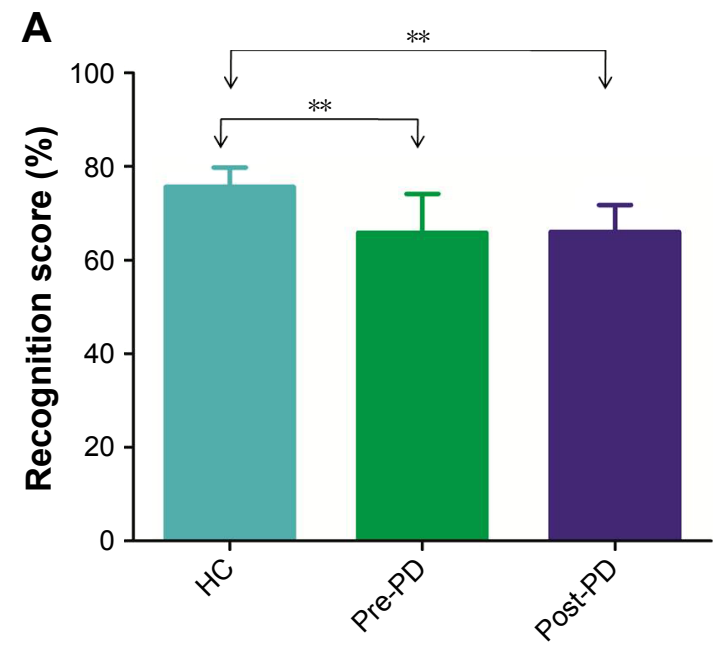

\section{Results \\ Emotional prosody recognition}

One-way ANOVA revealed a significant difference in total MAV scores among the three groups $(F[2,44]=11.018$, $P<0.001)$. Post hoc LSD tests revealed significantly higher total MAV scores in the HC group than in the pre-DBS $(P<0.001)$ and post-DBS $(P<0.001)$ PD groups (Figure $1 \mathrm{~A})$. ANOVA revealed significant effects of the factors "group" $(F[2,99]=3.861, P=0.023)$ and "emotion" $(F[4,99]=39.133$, $P<0.001)$ but did not identify a significant interaction between these two factors. Post hoc LSD tests revealed significantly lower MAV subscores for the neutral emotion in the pre-DBS and post-DBS PD groups than in the $\mathrm{HC}$ group $(P<0.001)$ (Figure 1B). There were no other significant differences observed between any two pairs of groups (all $P>0.05$ ).

\section{Emotional prosody expression}

In our analyses of accuracy rates, one-way ANOVA revealed significant differences among the three groups $(F[2,44]=$ 68.736, $P<0.001)$. Post hoc LSD tests showed significant differences between each pair of groups (Figure 2A). ANOVA revealed a significant main effect of the factors group $(F[2,224]=8.140, P<0.001)$ and emotion $(F[4,224]=22.064$, $P<0.001$; Figure 2B) but did not reveal a significant interaction between these two factors. Post hoc LSD tests revealed that prosody expressions of neutral emotions and anger were less well-recognized by the pre-DBS PD group than the HC group $(P<0.05)$. Fear expression was less well-recognized

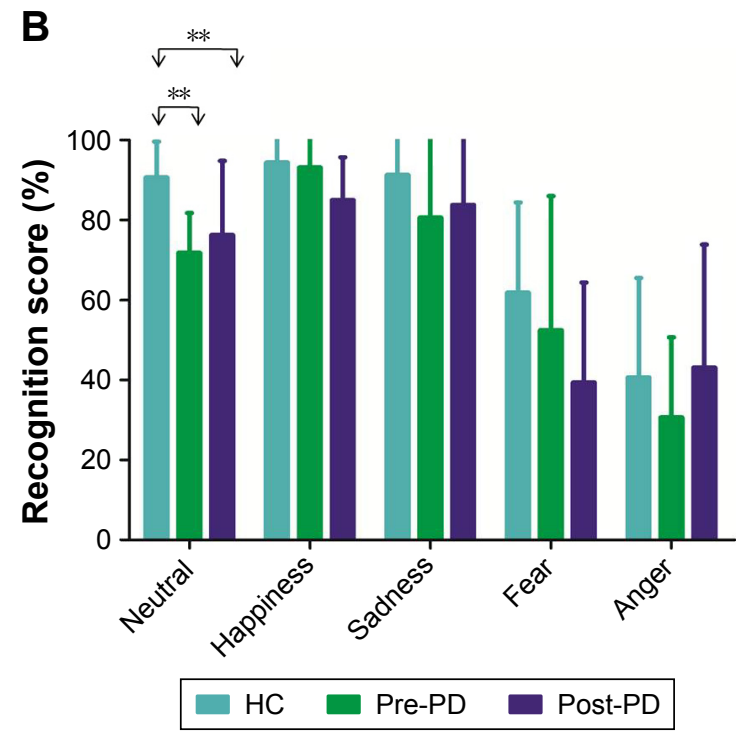

Figure I Emotional prosody recognition task for the HC group (blue), the pre-PD group (green), and the post-PD group (purple).

Notes: (A) Total score for all emotions and (B) sub-scores for single emotions. Data given are mean $\pm \mathrm{SD}$. **Significant if $P$-value $<0.01$.

Abbreviations: $\mathrm{HC}$, healthy control; PD, Parkinson's disease. 

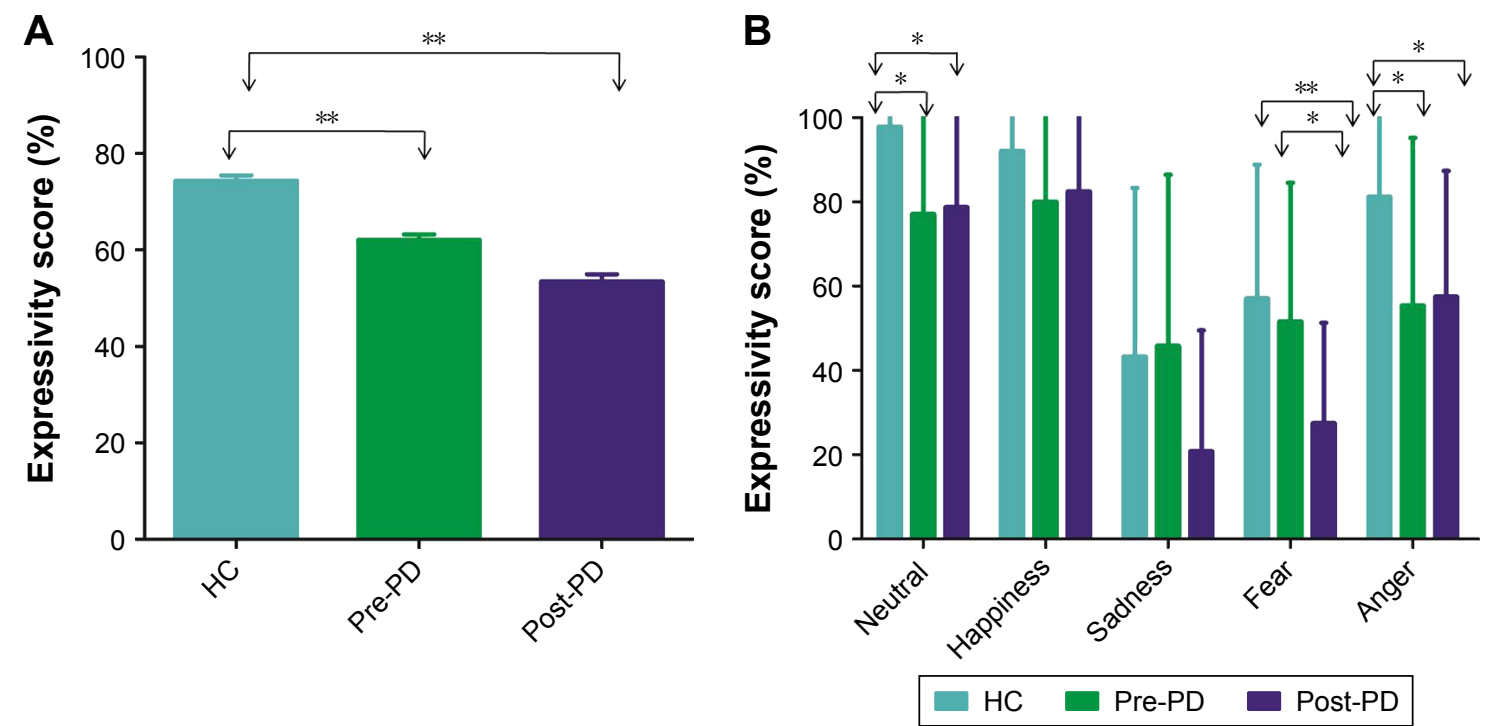

Figure 2 Emotional prosody expression task for the HC group (blue), the pre-PD group (green), and the post-PD group (purple).

Notes: (A) Total score for all emotions and (B) subscores for single emotions. Data given are mean $\pm \mathrm{SD}$. *Significant if $P$-value $<0.05$. **Significant if $P$-value $<0.01$.

Abbreviations: $\mathrm{HC}$, healthy control; PD, Parkinson's disease.

by the post-DBS PD group than the pre-DBS and HC groups. No other significant differences were observed between pairs of groups $(P>0.05)$.

RT and CL were analyzed using $\chi^{2}$ tests because the normality and homogeneity of variance assumptions were not satisfied. Significant differences in the distributions of RT $\left(\chi^{2}[2]=22.266, P<0.001\right.$; Figure $\left.3 \mathrm{~A}\right)$ and $\mathrm{CL}$ $\left(\chi^{2}[2]=828.151, P<0.001\right.$; Figure 4A) were observed among the three groups. Post hoc Mann-Whitney $U$ tests were used to identify differences in RT (Figure 3B) and CL (Figure 4B) among the groups. The following additional analogous statistical analyses were performed to compare the performance of the three groups on tests of each emotional category as measured by RT and CL: the $\chi^{2}$ test for three groups and the post hoc Mann-Whitney $U$ test for each pair of groups.

For mean pitch, we observe main effects for the factors group $(F[2,224]=4.195, P=0.016$; Figure $5 \mathrm{~A})$ and emotion $(F[4,224]=4.285, P=0.002$; Figure 5B). These results were significantly different. However, for the mean intensity, the results for the factors group $(F[2,224]=1.216, P=0.298$;
A

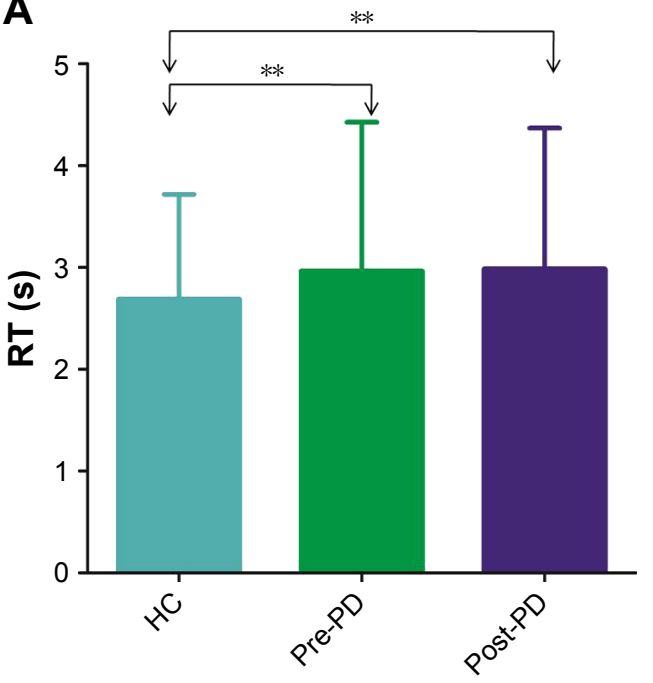

B

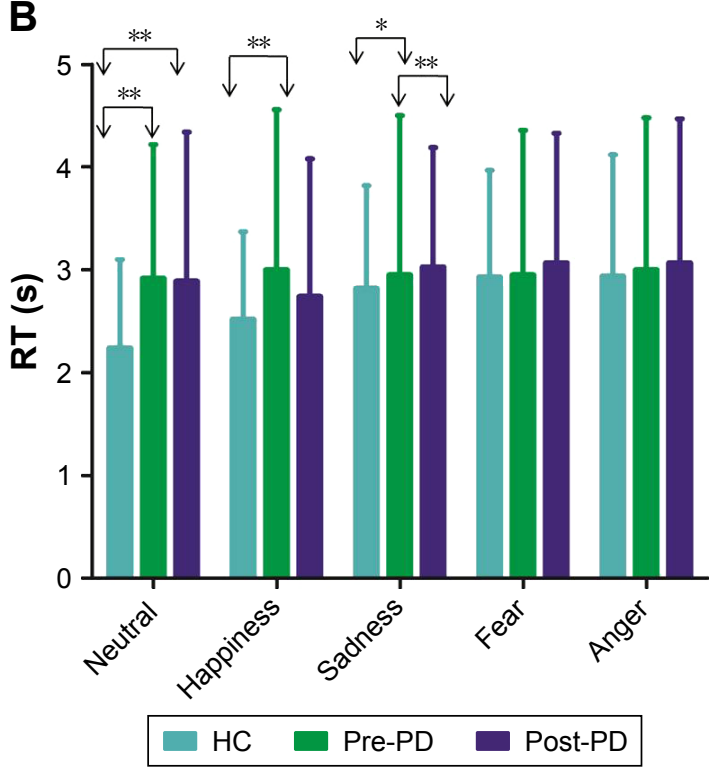

Figure 3 RT in the emotional prosody expression task for the HC group (blue), the pre-PD group (green), and the post-PD group (purple). Notes: (A) All emotions and (B) single emotions. Data given are mean \pm SD. *Significant if $P$-value $<0.05$. **Significant if $P$-value $<0.01$. Abbreviations: $\mathrm{HC}$, healthy control; PD, Parkinson's disease; RT, reaction time. 

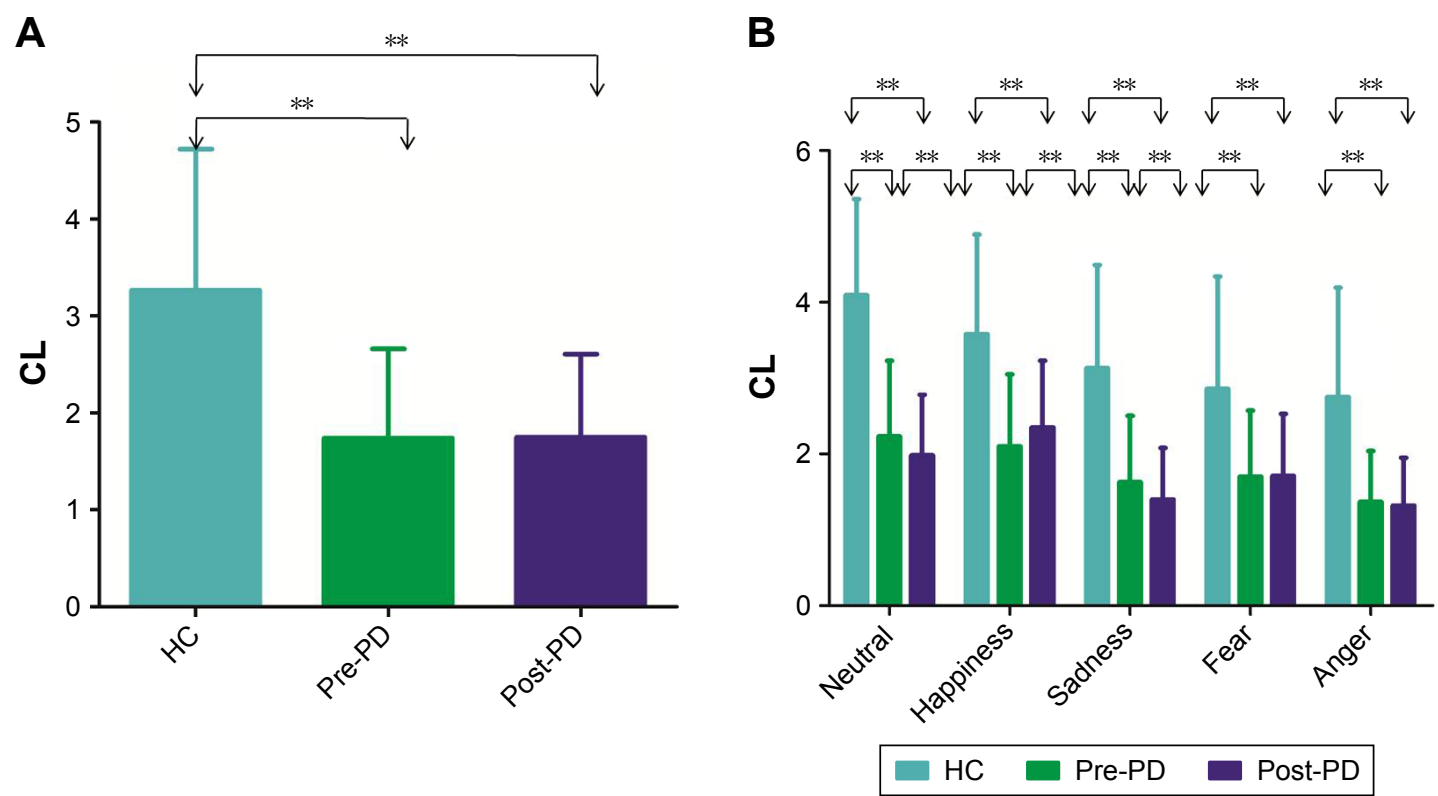

Figure $4 \mathrm{CL}$ in the emotional prosody expression task for the $\mathrm{HC}$ group (blue), the pre-PD group (green), and the post-PD group (purple).

Notes: (A) All emotions and (B) single emotions. Data given are mean \pm SD. **Significant if $P$-value $<0.01$.

Abbreviations: CL, confidence level; HC, healthy control; PD, Parkinson's disease.

Figure 6A) and emotion $(F[4,224]=10.484, P<0.001$; Figure 6B) were not significantly different. Post hoc LSD tests were performed to compare each pair of emotional categories.

\section{Correlations}

Pearson's correlation tests revealed a significant correlation between performance on emotional prosody recognition tests and performance on emotional prosody expression tests in the PD group ( $r=0.802, P<0.001)$ and the pre-DBS PD $(r=0.913, P<0.001)$ and post-DBS PD $(r=0.898, P<0.001)$ groups (Figure 7). The results are shown in Table 3. A weak correlation was observed between performance on emotional prosody expression tests and acoustic parameters, including the mean pitch and mean intensity. The results are shown in Table 4.

\section{Discussion}

The aim of this study was to confirm whether STN DBS influences emotional prosody processing in patients with PD.

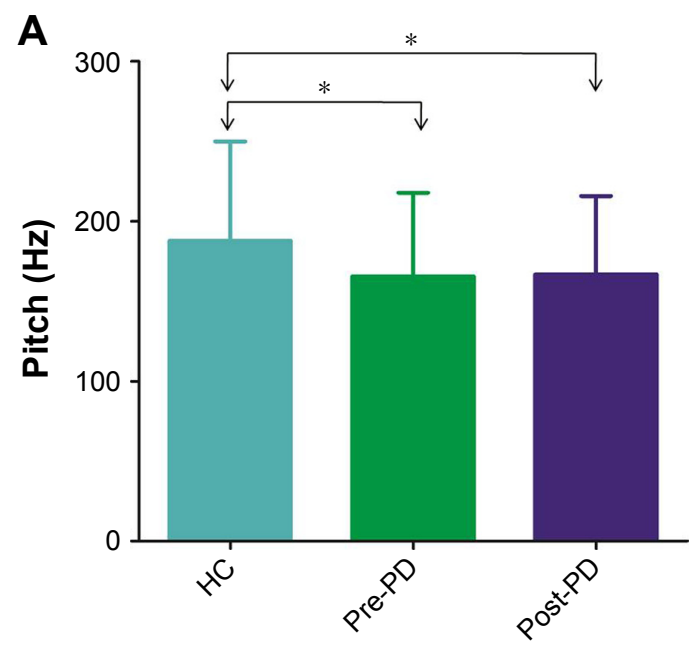

B

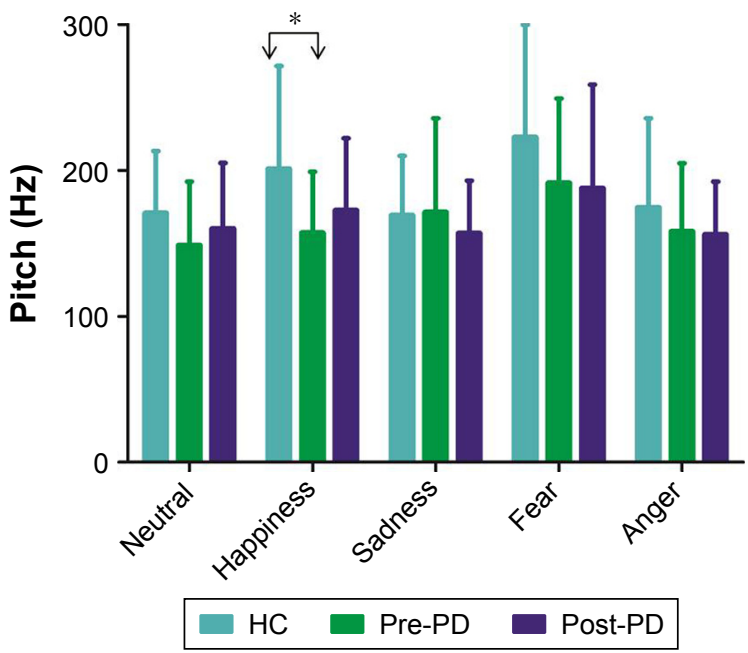

Figure 5 Pitch in the emotional prosody expression task for the HC group (blue), the pre-PD group (green), and the post-PD group (purple).

Notes: (A) All emotions and (B) single emotions. Data given are mean \pm SD. *Significant if $P$-value $<0.05$.

Abbreviations: HC, healthy control; PD, Parkinson's disease. 

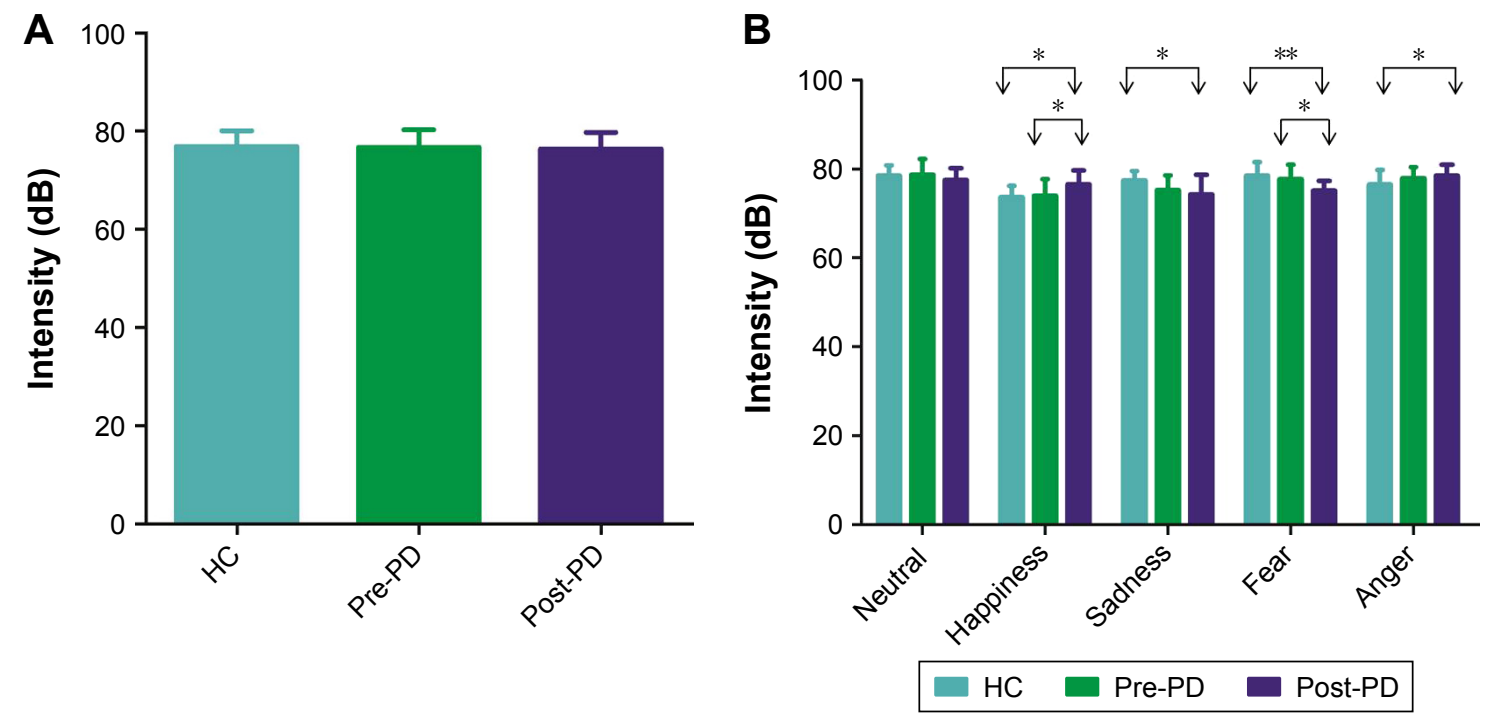

Figure 6 Intensity in the emotional prosody expression task for the HC group (blue), the pre-PD group (green), and the post-PD group (purple). Notes: (A) All emotions and (B) single emotions. Data given are mean \pm SD. *Significant if $P$-value $<0.05$. **Significant if $P$-value $<0.01$. Abbreviations: HC, healthy control; PD, Parkinson's disease.

We explored performance on emotional prosody recognition and expression tests by comparing selected parameters, such as the accuracy rate, RT, CL, and acoustic parameters, among pre-DBS, post-DBS, and HC groups ( $\mathrm{n}=16$ in each group).
Emotional prosody recognition, particularly the recognition of neutral emotions, was impaired to a greater extent in patients with PD than in HCs. These changes were attributed to the characteristic pathological changes that occurred in
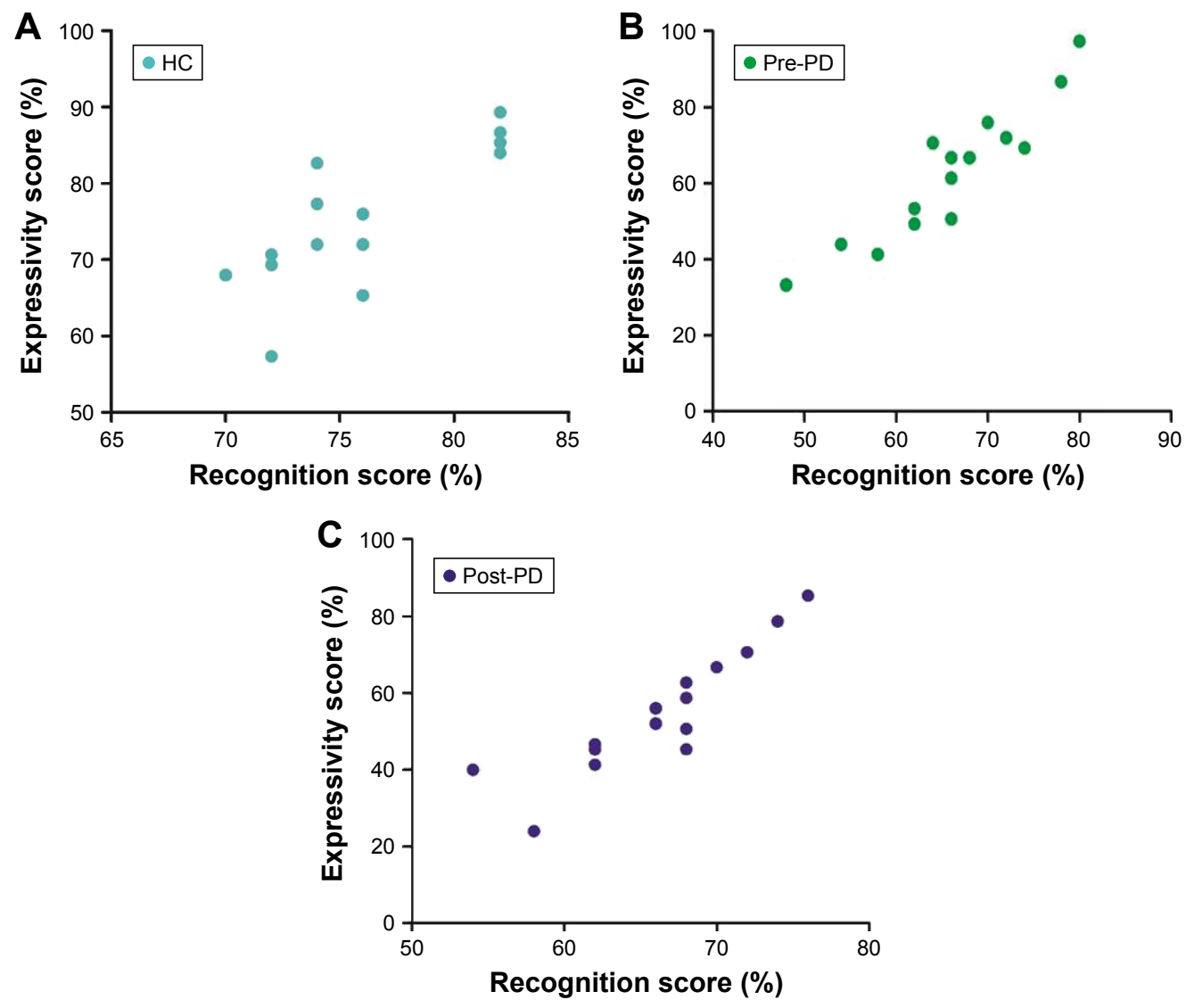

Figure 7 Correlation between emotional prosody recognition and emotional prosody expression for the HC (A), Pre-PD (B), and Post-PD (C) groups. Abbreviations: $\mathrm{HC}$, healthy control; PD, Parkinson's disease. 
Table 3 Correlation between emotional prosody recognition and expression

\begin{tabular}{lll}
\hline Groups & Pearson's $\boldsymbol{r}$ & P-values \\
\hline HC $(n=16)$ & 0.802 & 0.000 \\
Pre-PD $(n=16)$ & 0.913 & 0.000 \\
Post-PD $(n=16)$ & 0.898 & 0.000 \\
\hline
\end{tabular}

Abbreviations: HC, healthy control; PD, Parkinson's disease.

specific brain areas, such as the amygdala, thalamus, and prefrontal cortex. ${ }^{36-39}$ However, when we compared emotional prosody recognition between the pre-DBS and postDBS groups, we did not observe a significant reduction in patients with PD at 6 months after STN-DBS, consistent with the findings reported by Albuquerque et $\mathrm{al}^{24}$ and McIntosh et $\mathrm{al}^{40}$ and inconsistent with the finding reported by Péron et al. ${ }^{20}$ The discrepancies between these results likely result from the use of different methodologies (eg, the study design and case selection protocol) and the presence of confounding factors (such as cognitive impairment and a depressive state). The mean interval between DBS surgery and emotional assessments in the study performed by Péron et al was 35 ( $\mathrm{SD}=19.7$, range 3-72) months, which is not what would normally be described as "shortly" after surgery. However, the interval was 12 months in the study by Albuquerque et al, 3 months in the study by McIntosh et al, and 6 months in the present study. The prevalence of dementia was reported to steadily increase after DBS surgery in parallel with PD progression and could represent an early event. ${ }^{41}$ In addition, according to Péron et al, a higher Montgomery-Asberg Depression Rating Scale scores in the PD groups indicated the presence of more severe depression in patients with $\mathrm{PD}$ than in HC. However, we did not observe differences in neuropsychological characteristics among the three groups (Table 1).

Although the visual recognition of fear emotion was impaired by STN DBS in previous studies, no studies have explored emotional prosody expression processing in patients with PD who underwent STN DBS. Unlike emotional prosody recognition in this study, we provide the first evidence that the accuracy rate of identifying fear prosody expressions was altered after STN DBS. Notably, all three groups were

Table 4 Correlation between emotional prosody expression and acoustic parameters

\begin{tabular}{llllll}
\hline Groups & Pitch & & & Intensity & \\
\cline { 2 - 3 } & Pearson's $\boldsymbol{r}$ & P-values & & Pearson's $\boldsymbol{r}$ & P-values \\
\hline HC $(\mathrm{n}=16)$ & 0.353 & 0.180 & & 0.603 & 0.013 \\
Pre-PD $(\mathrm{n}=16)$ & 0.347 & 0.188 & & 0.011 & 0.967 \\
Post-PD $(\mathrm{n}=16)$ & 0.103 & 0.705 & & 0.578 & 0.019 \\
\hline
\end{tabular}

Abbreviations: $\mathrm{HC}$, healthy control; PD, Parkinson's disease. matched for demographic characteristics and the two PD groups were matched for clinical characteristics, indicating that neither demographic characteristics nor clinical characteristics explain the observed difference. Dopamine has been suggested to affect emotional processes. ${ }^{42,43}$ However, a significant difference in the use of dopamine replacement therapy was not observed between the two PD groups, suggesting that dopamine also does not explain the altered emotional expression observed in these patients. Thus, we propose that the modifications in the perception of emotional prosody expression that were identified in this study may be linked to a disturbance in the limbic territory of the STN. Intraoperative microelectrodes placed during DBS surgery recorded neuronal activity that was evoked by emotive auditory stimulation in the ventral nonoscillatory regions of the right STN, suggesting that the right ventral STN is potentially associated with the altered emotional effects on patients with PD after STN DBS observed in this study. ${ }^{44}$

Because the STN is small in size, intraoperative placement of electrodes in this nucleus must be precise. According to Witt et al, the best path to implant electrodes is from outside the caudate nuclei, because this path avoids the risk of causing a global cognitive deficit or affecting working memory performance after STN DBS. ${ }^{45}$ A series of advanced technologies, such as the multichannel microelectrode technique, $7 \mathrm{~T}$ high-field MRI, and iMRI, have been adopted at our center. The application of these techniques not only helps us to more accurately position electrodes during the preoperative and intraoperative stages but also allows us to explore the functions of different areas within this nucleus and other structures in the brain network.

In addition to evaluating the accuracy rate of prosody expression, we recorded the RT and CL of the raters. In the perception test, Cronbach's alpha was always $>0.90$ and the raters were blinded to the diagnoses. Hence, the results of the perception test had high inter-rater reliability. Compared to the $\mathrm{HC}$ group, the PD groups required longer times to identify the recorded vocal materials and felt less confident in their choices.

Objective methods were also included; for example, we analyzed two acoustic parameters (mean pitch and mean intensity) of the recorded materials to explore whether the parameters are useful for distinguishing emotional speech in the three groups. The speech of the patients with PD displayed a lower volume, a nonemotional pitch, and an abnormal speech rate. Several acoustic parameters are recommended for use in patients with an early PD diagnosis and while monitoring disease progression. ${ }^{6}$ Although no significant differences in mean intensity were observed 
among the groups, a significant difference in the mean pitch was observed among the groups. In addition, we identified weak correlations between performance on emotional prosody expression tests and acoustic parameters. Future studies should include additional acoustic parameters when evaluating the abilities of PD and $\mathrm{HC}$ groups to distinguish prosody expression.

Another novel finding in this study was the significant correlation between performance on emotional prosody recognition tests and performance on emotional prosody expression tests in both the pre-DBS and post-DBS PD groups. A "shared models" hypothesis is therefore suggested. According to this hypothesis, an emotion can stimulate same emotional state when an individual recognizes the emotion expressed by others because a large number of similar brain areas are activated when observing and imitating emotional expression. ${ }^{46,47}$

We acknowledge that this study has certain limitations. First, a rather small number of participants were included and other factors, such as age, gender, dopamine replacement therapy, and disease stages, were not investigated. This study is explorative in nature, and multiple comparisons should be performed in further studies. Second, a restricted number of emotional categories were used. Changes in other emotional categories may have been missed in our post-STN DBS group. Third, only two acoustic parameters were included. We are prepared to examine additional acoustic parameters including pitch and intensity variations and verbal or nonverbal sentences in further studies. ${ }^{23,35}$ Fourth, we used posed prosody expressions rather than spontaneous expressions in these tests. The mechanisms underlying the responses to these two types of expressions may differ. Fifth, functional imaging was not conducted and we were therefore unable to correlate behavioral results with activity in specific brain areas. Sixth, we did not investigate the changes in emotional prosody in the same pre-DBS and post-DBS patients but examined them in different patient groups. We acknowledge that a better comparison would be to use the same pre- and post-DBS patients. However, consistent with the findings reported by McIntosh et $\mathrm{al}^{40}$ and Péron et al, ${ }^{20}$ we did not observe differences in the neuropsychological characteristics, demographic characteristics, or clinical data among the two groups (Table 1), although different patients were included in each group.

\section{Conclusion}

Based on the results of our study, the ability to recognize emotional prosody was not altered but fear expression was impaired shortly after STN DBS. Abnormalities in emotional prosody recognition and expression deficits were associated with time points both before and after STN DBS, indicating that emotional prosody recognition and expression may share common neuronal mechanisms. More acoustic parameters that can distinguish prosody expression between $\mathrm{PD}$ and $\mathrm{HC}$ groups should be analyzed in future studies.

\section{Disclosure}

The authors report no conflicts of interest in this work.

\section{References}

1. Verhagen ML, Pal G, Slavin K. Surgical treatment of Parkinson's disease. Curr Treat Options Neurol. 2016;18(11):49.

2. Troche MS, Brandimore AE, Foote KD, et al. Swallowing outcomes following unilateral STN vs. GPi surgery: a retrospective analysis. Dysphagia. 2014;29(4):425-431.

3. Williams NR, Foote KD, Okun MS. STN vs. GPi deep brain stimulation: translating the rematch into clinical practice. Mov Disord Clin Pract. 2014;1(1):24-35.

4. Sidiropoulos C, LeWitt PA, Odekerken VJ, Schuurman PR, de Bie RM. GPi vs STN deep brain stimulation for Parkinson disease: three-year follow-up. Neurology. 2016;87(7):745-746.

5. Péron J, Cekic S, Haegelen C, et al. Sensory contribution to vocal emotion deficit in Parkinson's disease after subthalamic stimulation. Cortex. 2015;63:172-183.

6. Brabenec L, Mekyska J, Galaz Z, Rektorova I. Speech disorders in Parkinson's disease: early diagnostics and effects of medication and brain stimulation. J Neural Transm (Vienna). 2017;124(3):303-334.

7. Chen X, Pan Z, Wang P, et al. The integration of facial and vocal cues during emotional change perception: EEG markers. Soc Cogn Affect Neurosci. 2016;11(7):1152-1161.

8. Grandjean D, Bänziger T, Scherer KR. Intonation as an interface between language and affect. Prog Brain Res. 2006;156:235-247.

9. Witteman J, Van Heuven VJ, Schiller NO. Hearing feelings: a quantitative meta-analysis on the neuroimaging literature of emotional prosody perception. Neuropsychologia. 2012;50(12):2752-2763.

10. Ethofer T, Anders S, Erb M, et al. Cerebral pathways in processing of affective prosody: a dynamic causal modeling study. Neuroimage. 2006;30(2):580-587.

11. Brück C, Wildgruber D, Kreifelts B, Krüger R, Wächter T. Effects of subthalamic nucleus stimulation on emotional prosody comprehension in Parkinson's disease. PLoS One. 2011;6(4):e19140.

12. Belin P, Zatorre RJ. 'What', 'where' and 'how' in auditory cortex. Nat Neurosci. 2000;3(10):965-966.

13. Grandjean D, Sander D, Pourtois G, et al. The voices of wrath: brain responses to angry prosody in meaningless speech. Nat Neurosci. 2005;8(2):145-146.

14. Ethofer T, Bretscher J, Gschwind M, Kreifelts B, Wildgruber D, Vuilleumier P. Emotional voice areas: anatomic location, functional properties, and structural connections revealed by combined fMRI/DTI. Cereb Cortex. 2012;22(1):191-200.

15. Frühholz S, Grandjean D. Processing of emotional vocalizations in bilateral inferior frontal cortex. Neurosci Biobehav Rev. 2013; 37(10 pt 2):2847-2855.

16. Ceravolo L, Frühholz S, Grandjean D. Modulation of auditory spatial attention by angry prosody: an fMRI auditory dot-probe study. Front Neurosci. 2016;10:216.

17. Liebenthal E, Silbersweig DA, Stern E. The language, tone and prosody of emotions: neural substrates and dynamics of spoken-word emotion perception. Front Neurosci. 2016;10:506. 
18. Mitchell RL, Jazdzyk A, Stets M, Kotz SA. Recruitment of language-, emotion- and speech-timing associated brain regions for expressing emotional prosody: investigation of functional neuroanatomy with fMRI. Front Hum Neurosci. 2016;10:518.

19. Simon D, Becker M, Mothes-Lasch M, Miltner WH, Straube T. Loud and angry: sound intensity modulates amygdala activation to angry voices in social anxiety disorder. Soc Cogn Affect Neurosci. 2017;12(3): 409-416.

20. Péron J, Grandjean D, Le JF, et al. Recognition of emotional prosody is altered after subthalamic nucleus deep brain stimulation in Parkinson's disease. Neuropsychologia. 2010;48(4):1053-1062.

21. Pell MD, Cheang HS, Leonard CL. The impact of Parkinson's disease on vocal-prosodic communication from the perspective of listeners. Brain Lang. 2006;97(2):123-134.

22. Dara C, Monetta L, Pell MD. Vocal emotion processing in Parkinson's disease: reduced sensitivity to negative emotions. Brain Res. 2008; 1188:100-111.

23. Rusz J, Cmejla R, Ruzickova H, Ruzicka E. Quantitative acoustic measurements for characterization of speech and voice disorders in early untreated Parkinson's disease. J Acoust Soc Am. 2011;129(1): 350-367.

24. Albuquerque L, Coelho M, Martins M, Martins IP. STN-DBS does not change emotion recognition in Parkinson's disease. Parkinsonism Relat Disord. 2014;20(5):564-565.

25. Péron J. Does STN-DBS really not change emotion recognition in Parkinson's disease. Parkinsonism Relat Disord. 2014;20(5):562-563.

26. Monetta L, Cheang HS, Pell MD. Understanding speaker attitudes from prosody by adults with Parkinson's disease. J Neuropsychol. 2008; 2(pt 2):415-430

27. Calvo MG, Lundqvist D. Facial expressions of emotion (KDEF): identification under different display-duration conditions. Behav Res Methods. 2008;40(1):109-115.

28. Keshtiari N, Kuhlmann M, Eslami M, Klann-Delius G. Recognizing emotional speech in Persian: a validated database of Persian emotional speech (Persian ESD). Behav Res Methods. 2015;47(1):275-294.

29. Benabid AL, Pollak P, Gross C, et al. Acute and long-term effects of subthalamic nucleus stimulation in Parkinson's disease. Stereotact Funct Neurosurg. 1994;62(1-2):76-84.

30. Benabid AL, Krack PP, Benazzouz A, Limousin P, Koudsie A, Pollak P. Deep brain stimulation of the subthalamic nucleus for Parkinson's disease: methodologic aspects and clinical criteria. Neurology. 2000; 55(12 suppl 6):S40-S44

31. Cui Z, Pan L, Song H, et al. Intraoperative MRI for optimizing electrode placement for deep brain stimulation of the subthalamic nucleus in Parkinson disease. J Neurosurg. 2016;124(1):62-69.

32. Belin P, Fillion-Bilodeau S, Gosselin F. The Montreal Affective Voices: a validated set of nonverbal affect bursts for research on auditory affective processing. Behav Res Methods. 2008;40(2):531-539.
33. Banse R, Scherer KR. Acoustic profiles in vocal emotion expression. J Pers Soc Psychol. 1996;70(3):614-636.

34. Liu P, Pell MD. Recognizing vocal emotions in Mandarin Chinese: a validated database of Chinese vocal emotional stimuli. Behav Res Methods. 2012;44(4):1042-1051.

35. Tykalova T, Rusz J, Cmejla R, Ruzickova H, Ruzicka E. Acoustic investigation of stress patterns in Parkinson's disease. J Voice. 2014; 28(1):e129.e1-e129.e8.

36. Alexander GE, Crutcher MD. Functional architecture of basal ganglia circuits: neural substrates of parallel processing. Trends Neurosci. 1990;13(7):266-271.

37. Alexander GE, DeLong MR, Strick PL. Parallel organization of functionally segregated circuits linking basal ganglia and cortex. Annu Rev Neurosci. 1986;9:357-381.

38. Middleton FA, Strick PL. Basal-ganglia 'projections' to the prefrontal cortex of the primate. Cereb Cortex. 2002;12(9):926-935.

39. Le JF, Péron J, Biseul I, et al. Subthalamic nucleus stimulation affects orbitofrontal cortex in facial emotion recognition: a PET study. Brain 2008;131(pt 6):1599-1608.

40. McIntosh LG, Mannava S, Camalier CR, et al. Emotion recognition in early Parkinson's disease patients undergoing deep brain stimulation or dopaminergic therapy: a comparison to healthy participants. Front Aging Neurosci. 2014;6:349.

41. Bang HM, Johnsen EL, Sunde N, Vase A, Gjelstrup MC, Østergaard K. Surviving 10 years with deep brain stimulation for Parkinson's diseasea follow-up of 79 patients. Eur J Neurol. 2016;23(1):53-61.

42. Skodda S, Visser W, Schlegel U. Short- and long-term dopaminergic effects on dysarthria in early Parkinson's disease. J Neural Transm (Vienna). 2010;117(2):197-205.

43. Rusz J, Tykalová T, Klempír J, Čmejla R, Růžička E. Effects of dopaminergic replacement therapy on motor speech disorders in Parkinson's disease: longitudinal follow-up study on previously untreated patients. J Neural Transm (Vienna). 2016;123(4):379-387.

44. Eitan R, Shamir RR, Linetsky E, et al. Asymmetric right/left encoding of emotions in the human subthalamic nucleus. Front Syst Neurosci. 2013;7:69.

45. Witt K, Granert O, Daniels C, et al. Relation of lead trajectory and electrode position to neuropsychological outcomes of subthalamic neurostimulation in Parkinson's disease: results from a randomized trial. Brain. 2013;136(pt 7):2109-2119.

46. Carr L, Iacoboni M, Dubeau MC, Mazziotta JC, Lenzi GL. Neural mechanisms of empathy in humans: a relay from neural systems for imitation to limbic areas. Proc Natl Acad Sci U S A. 2003;100(9):5497-5502.

47. Ricciardi L, Visco-Comandini F, Erro R, et al. Facial emotion recognition and expression in Parkinson's disease: an emotional mirror mechanism. PLoS One. 2017;12(1):e0169110.
Neuropsychiatric Disease and Treatment

\section{Publish your work in this journal}

Neuropsychiatric Disease and Treatment is an international, peerreviewed journal of clinical therapeutics and pharmacology focusing on concise rapid reporting of clinical or pre-clinical studies on a range of neuropsychiatric and neurological disorders. This journa is indexed on PubMed Central, the 'PsycINFO' database and CAS,

\section{Dovepress}

and is the official journal of The International Neuropsychiatric Association (INA). The manuscript management system is completely online and includes a very quick and fair peer-review system, which is all easy to use. Visit http://www.dovepress.com/testimonials.php to read real quotes from published authors. 